\title{
Tuned Gyro-Pendulum Stabilizer for Control of Vibrations in Structures
}

\author{
Faruk Ünker \\ Department of Mechanical Engineering, Gümüşhane University, 29100 Gümüşhane, Turkey.
}

\begin{abstract}
(Received 22 June 2019; accepted 17 November 2019)
\end{abstract}
A special combined gyro-pendulum stabilizer (a gyroscope with coupling to a pendulum) mounted on a vibrating mass is considered for investigation of the vibration responses. This paper mainly focuses on the derivation of the frequency equations and on finding the required angular momentum for vibration control of the system. Besides, there is also an ANSYS simulation model of gyro-pendulum, which was built to verify the mathematical model. The dynamic responses of both that obtained from ANSYS simulation and that obtained from numerical solving of a Lagrangian mathematical model are analyzed comparatively. The angular momentum $\left(\Omega I_{p}\right)$, in relation to the natural frequency $\left(\omega_{n}\right)$ of the primary mass, shows that this vibration control device is more adaptable than other conventional ones by producing unidirectional thrust along the forcing excitation axis whilst the gyroscope is spinning.

\section{INTRODUCTION}

Vibrations are present in many different mechanical systems and may cause undesirable motion and sometimes damage to the mechanical and structural systems. So the effective attenuating of mechanical vibrations has always been a big challenge for engineers. Nowadays, there are different ways of mitigating vibrations by applying one or more passive dynamic vibration absorbers. Passive vibration absorbers have the advantage of not requiring external force or energy. ${ }^{1-3}$ The passive control method is activated by the structural motion without requiring external force or energy to reduce structural responses and utilizes the motion of the structure at the location of the device. ${ }^{4}$ The most widely used passive vibration absorbers in engineering are tuned mass absorbers, beam absorbers, pendulum-type absorbers, and even liquid absorbers. Tuned mass dampers (TMDs) are widely used for passive vibration mitigation of mechanical structures due to their simple structure. ${ }^{5-10}$ One of the first attempts, known as the tuned mass damper (TMD), consists of a mass-spring system with no damping, which was introduced by Hermann Frahm in 1911. As it is well known, classic TMD is tuned to the structural fundamental frequency such that the natural frequency of the absorber is equal to the target frequency to be effective in reducing the primary structure's response. So, the TMD is a single-frequency vibration absorber that only works within a narrow frequency band and is susceptible to the frequency ratio. However, adding a damper to TMDs has been presented in the work of Ormondroyd and Den Hartog (1928) to have a better application for a wide frequency bandwidth. ${ }^{11}$ The authors propose that the addition of the viscous damper to Frahm's system design can expand the effective frequency band around the principal resonance.

Pendulum vibration absorbers (PVA) can be utilized as TMD to attenuate unfavorable motions of subjected systems and commonly used to control horizontal, vertical and rotational vibrations of main structure because PVAs can oscillate regardless of the direction of base structure motion., ${ }^{2,3}$ Moreover, PVAs have low frequency and use gravitational force instead of elastic stiffness force, so they are widely used in practice for reducing the level of vibrations of buildings or bridges. $^{12}$ PVAs can be quite effective and desirable due to their simplicity and lower cost.

In recent years, several research techniques have been used to conduct studies investigating the complicated motions which appear in a gyrostabilizer. ${ }^{13-15}$ The gyroscopic moment induced by a rotating object offers an attractive means to protect structures against natural hazards in various ways. ${ }^{14}$ Compared to conventional active mass dampers for seismic vibration suppression, gyrostabilizers represent a weight and volume saving. The use of gyrostabilizers has emerged, as they have an ability to control vibration at low frequency, and, furthermore, the stored kinetic energy can provide emergency power. ${ }^{13,15}$ The mechanism does not require any other external source of energy, in which the rotor speed is produced by an electric motor in a rotating gimbal. Therefore, this can be classified as a passive control device in a variety of passive vibration control systems.

In this paper, the performance of a gyroscope with a spherical PVA is investigated when attached to a structure that is modeled as a one-degree-of-freedom system under horizontal, harmonic excitation. Since the spherical pendulum can oscillate regardless of the direction of base structure motion, the gimbal of pendulum-like gyrostabilizer (gyro-pendulum) can precess by the rotating disk to convert the angular momentum to linear due to the gravitational force existing on the mass of the pendulum. Hence, the gyro-pendulum in the proposed configuration is supposed to be an effective device that can work in a wide range of excitation frequencies, while much easier changing the disk speed of gyroscope to tune the mode frequencies on the main resonant frequency of the primary system. The classical PVAs have low frequency, and their effectiveness is strongly limited by the energy transfer from the primary system to the PVAs. However, the gyro-pendulum vibration absorbers are much effective in a larger frequency range from pendulum resonance to main system resonance due to the angular momentum of the gyroscope. 


Table 1. Physical properties of the system.
\begin{tabular}{||l|l|l||}
\hline Symbol & Numerical values & Description \\
\hline$g$ & $9.81 \mathrm{~m} / \mathrm{s}^{2}$ & Gravitational acceleration \\
$L$ & $0.49766 \mathrm{~m}$ & Length of the centroid of the inner gimbal mass \\
$m_{s}$ & $99.997 \mathrm{~kg}$ & Mass of the inner gimbal \\
$m_{p}$ & $20 \mathrm{~kg}$ & Mass of the outer gimbal \\
$m_{d}$ & $10.001 \mathrm{~kg}$ & Disk mass of the gyroscope \\
$m_{c}$ & $8000.1 \mathrm{~kg}$ & Main mass \\
$L_{c}$ & $4 \mathrm{~m}$ & Length of the column \\
$h$ & $0.2 \mathrm{~m}$ & Column thickness \\
$b$ & $0.6 \mathrm{~m}$ & Column width \\
$E$ & $24 \times 10^{9} \mathrm{~N} / \mathrm{m}^{2}$ & Young's modulus of the column \\
$I_{c}=1 / 12 b h^{3}$ & $4 \times 10^{-4} \mathrm{~m}^{4}$ & Geometrical moment of inertia of the column \\
$k_{c}=3 E I_{c} / L_{c}^{3}$ & $4.5 \times 10^{5} \mathrm{~N} / \mathrm{m}$ & Stiffness of each column \\
$k=4 k_{c}$ & $18 \times 10^{5} \mathrm{~N} / \mathrm{m}$ & Equivalent stiffness of columns \\
$I_{p}$ & $0.20014 \mathrm{~kg} \mathrm{~m}{ }^{2}$ & The rotary inertia of the disk \\
$I_{o}$ & $0.10019 \mathrm{~kg} \mathrm{~m}{ }^{2}$ & Mass moment of inertia of the disk \\
$I_{f x}$ & $0.32202 \mathrm{~kg} \mathrm{~m}$ & Principal moments of inertia of the inner gimbal (Taken at the center of mass of the inner gimbal) \\
$I_{f y}$ & $9.98810 \mathrm{~kg} \mathrm{~m}{ }^{2}$ & Principal moments of inertia of the inner gimbal (Taken at the center of mass of the inner gimbal) \\
$I_{f z}$ & $9.85790 \mathrm{~kg} \mathrm{~m}{ }^{2}$ & Principal moments of inertia of the inner gimbal (Taken at the center of mass of the inner gimbal) \\
$I_{p y}$ & $0.67851 \mathrm{~kg} \mathrm{~m}{ }^{2}$ & Principal moments of inertia of the outer gimbal (Taken at the center of mass of the outer gimbal) \\
$\Omega$ & $0-50000 \mathrm{rpm}^{2}$ & Rotating speed of the disk \\
$z_{o}$ & $0.001 \mathrm{~m}$ & Harmonic disturbing amplitude \\
\hline
\end{tabular}

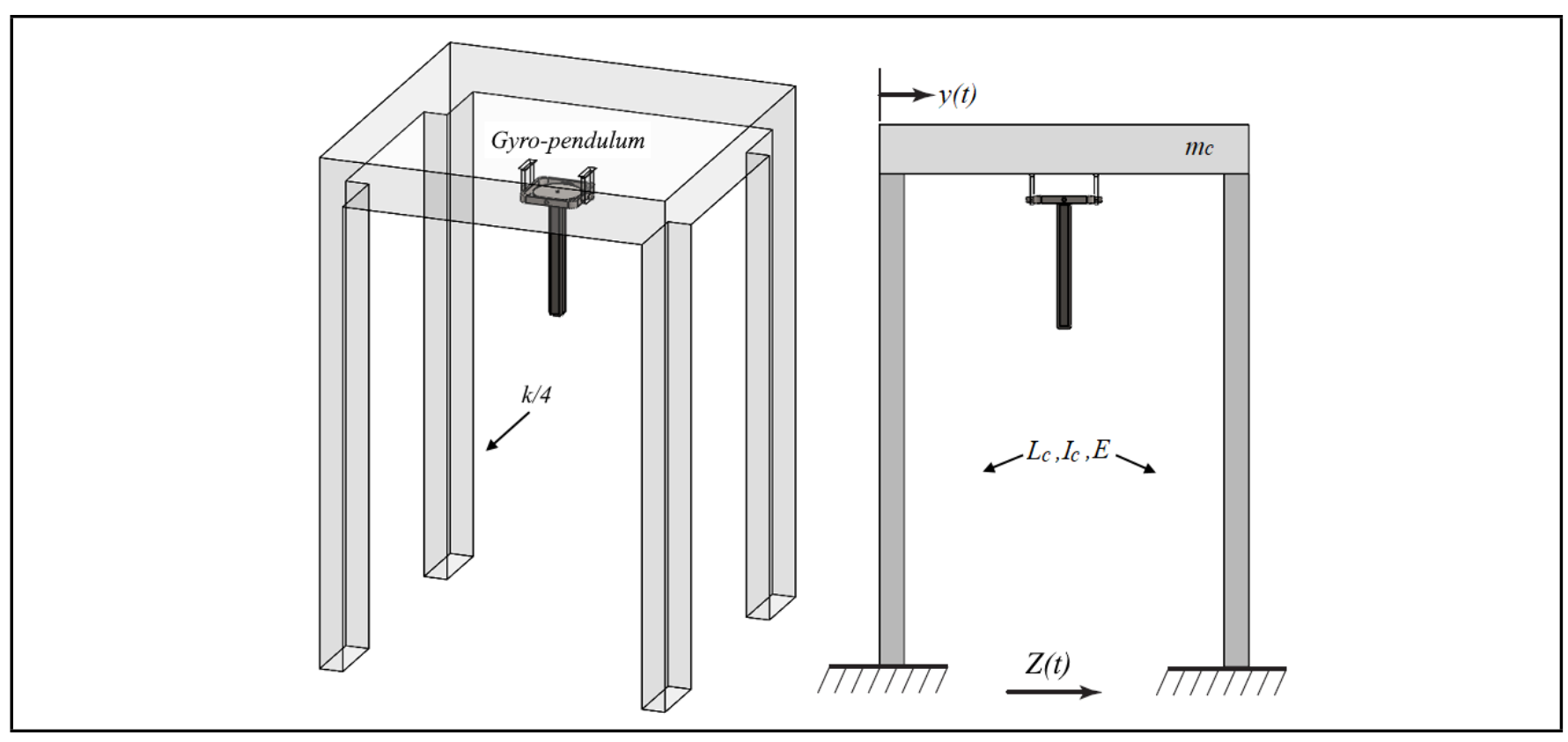

Figure 1. Physical model of the system.

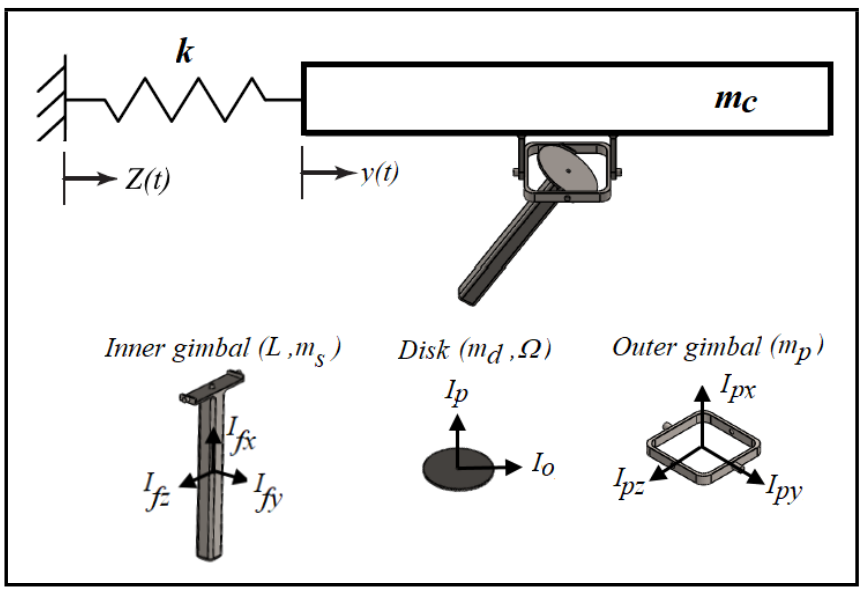

Figure 2. The reduced model of the physical system.

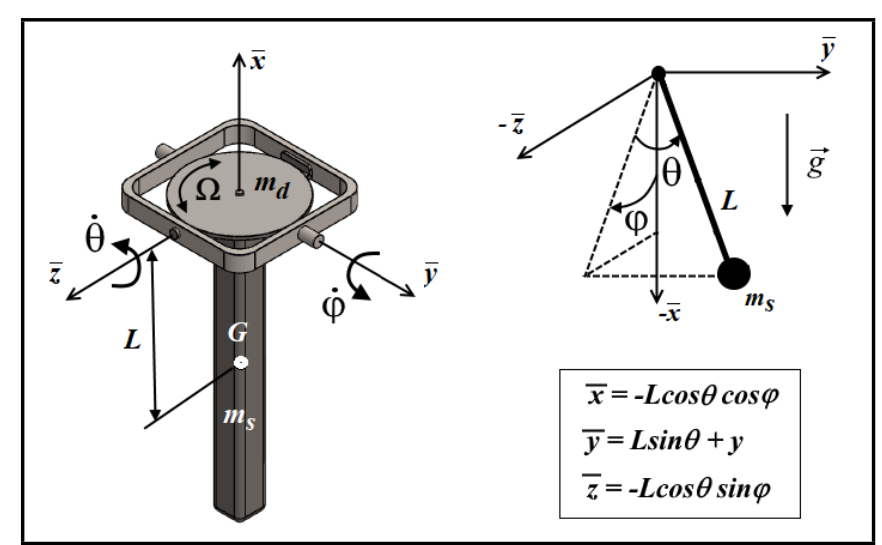

Figure 3. Gyro-pendulum via a pendulum arm of stabilizing mass, $m_{s}$ with length $L$ from the centroid $(G)$. 


\section{SYSTEM SET-UP}

The physical model of the system is shown in Fig. 1 and the physical properties of the system are given in Table 1 . Sinusoidal excitation is introduced horizontally to the base of the primary mass as shown in Figs. 1 and 2. The base of the spring-mass model harmonically excites with different forcing frequencies $(\omega)$ and forcing amplitudes $\left(z_{0}\right)$ with the horizontal base excitation of $Z=z_{0} \cos (\omega t)$. The horizontal elastic displacement at the free end is $y$. The gyro (the disk) is mounted in a gimbal of a pendulum with coupling to an outer gimbal as shown in Fig. 3. The gyro-pendulum system consists of a disk mass $m_{d}$, which can spin freely about its geometric axis (by an inner gimbal) mounted to the outer gimbal of the main mass. The disk of the gyro is assumed to have a rotational speed $\Omega$ and a precession $\varphi$.

\section{EQUATIONS OF MOTION OF GYRO- PENDULUM}

There is one spinning disk connected with a pendulum supported by the pivot axis of an outer gimbal which is positioned with respect to the main mass as shown in Fig. 2. As shown in Fig. 3, one gyroscopic disk is spinning at a constant speed to have precession $\varphi$ to compensate for the gravitational force existing on the inner gimbal due to the motion of the pendulum. The gyro-pendulum absorber can be assumed to have three degrees of freedom when the disk is spinning with constant angular velocity $\Omega$. Therefore, this paper is based on the equations of motion, written in the form of three differential equations (Eqs. (4), (5), and (6)) with three unknowns $(y, \varphi$, and $\theta$ ).

Here, the kinetic energies of the gyroscope (disk), inner gimbal, outer gimbal, and the main mass can be written as follows, respectively:

$$
\begin{aligned}
T_{\text {disk }}= & \frac{1}{2} m_{d} \dot{y}^{2}+\frac{1}{2} I_{o}\left(\dot{\theta}^{2}+\dot{\varphi}^{2} \cos ^{2} \theta\right)+ \\
& \frac{1}{2} I_{p}(\Omega+\dot{\varphi} \sin \theta)^{2} \\
T_{\text {inner_gimbal }}= & \frac{1}{2} m_{s}\left[(L \dot{\theta} \cos \theta+\dot{y})^{2}+(L \dot{\theta} \sin \theta)^{2}+\right. \\
& \left.(L \dot{\varphi} \cos \theta)^{2}\right]+\frac{1}{2} I_{f x} \dot{\varphi}^{2} \sin ^{2} \theta+ \\
& \frac{1}{2} I_{f y} \dot{\varphi}^{2} \cos ^{2} \theta+\frac{1}{2} I_{f z} \dot{\theta}^{2} \\
T_{\text {outer_gimbal }}= & \frac{1}{2} m_{p} \dot{y}^{2}+\frac{1}{2} I_{p y} \dot{\varphi}^{2} \\
T_{\text {main_mass }}= & \frac{1}{2} m_{c} \dot{y}^{2} .
\end{aligned}
$$

Therefore, the total kinetic and potential energies of the system can be expressed as follows, respectively:

$$
\begin{aligned}
& T_{\text {total }}=T_{\text {disk }}+T_{\text {inner_gimbal }}+T_{\text {outer_gimbal }}+T_{\text {main_mass }} ; \\
& V_{\text {total }}=-m_{s} g L \cos \theta \cos \varphi+\frac{1}{2} k(y-Z)^{2} .
\end{aligned}
$$

Then the equations of motion describing the gyro-pendulum can be obtained from the Lagrange equations. For this system, the Lagrange equations become:

$$
\begin{aligned}
& \frac{d}{d t}\left(\frac{\partial T_{\text {total }}}{\partial \dot{y}}\right)-\frac{\partial T_{\text {total }}}{\partial y}+\frac{\partial V_{\text {total }}}{\partial y}=0 ; \\
& \frac{d}{d t}\left(\frac{\partial T_{\text {total }}}{\partial \dot{\theta}}\right)-\frac{\partial T_{\text {total }}}{\partial \theta}+\frac{\partial V_{\text {total }}}{\partial \theta}=0 ; \\
& \frac{d}{d t}\left(\frac{\partial T_{\text {total }}}{\partial \dot{\varphi}}\right)-\frac{\partial T_{\text {total }}}{\partial \varphi}+\frac{\partial V_{\text {total }}}{\partial \varphi}=0 .
\end{aligned}
$$

Hence, by applying Lagrange's equations, the fundamental equations of the gyro-pendulum motion are obtained as:

$M_{t} \ddot{y}+\ddot{\theta} m_{s} L \cos \theta-\dot{\theta}^{2} m_{s} L \sin \theta+k(y-Z)=0 ;$

$\left(I_{o}+I_{f z}+m_{s} L^{2}\right) \ddot{\theta}+\left(\ddot{y} m_{s} L-I_{p} \Omega \dot{\varphi}\right) \cos \theta-\left(I_{p}-I_{o}+\right.$

$\left.I_{f x}-I_{f y}-m_{s} L^{2}\right) \dot{\varphi}^{2} \sin \theta \cos \theta+m_{s} g L \sin \theta \cos \varphi=0$

$\left(m_{s}(L \cos \theta)^{2}+I_{p y}+I_{o} \cos ^{2} \theta+I_{p} \sin ^{2} \theta+I_{f x} \sin ^{2} \theta+\right.$

$\left.I_{f y} \cos ^{2} \theta\right) \ddot{\varphi}+2\left(I_{p}-I_{o}+I_{f x}-I_{f y}-m_{s} L^{2}\right) \dot{\varphi} \dot{\theta} \sin \theta \cos \theta+$ $I_{p} \Omega \dot{\theta} \cos \theta+m_{s} g L \cos \theta \sin \varphi=0$;

in which

$$
M_{t}=m_{c}+m_{d}+m_{s}+m_{p}
$$

\subsection{Equations of Motion about the Equilibrium Position for Small Vibration}

The kinetic energy is zero when the primary mass $\left(M_{t}\right)$ is at the equilibrium position $(\dot{y}=0)$. So, the problem can be simplified by substituting $\dot{\theta} \approx 0$ and $\dot{\varphi} \approx 0$ in Eqs. (7), (8), and (9) near to the equilibrium position. Now that the motion can be reduced as:

$$
\begin{aligned}
& M_{t} \ddot{y}+\ddot{\theta} m_{s} L \cos \theta+k(y-Z)=0 \\
& \left(I_{o}+I_{f z}+m_{s} L^{2}\right) \ddot{\theta}+\left(\ddot{y} m_{s} L-I_{p} \Omega \dot{\varphi}\right) \cos \theta+ \\
& m_{s} g L \sin \theta \cos \varphi=0 \\
& \left(m_{s}(L \cos \theta)^{2}+I_{p y}+I_{o} \cos ^{2} \theta+I_{p} \sin ^{2} \theta+I_{f x} \sin ^{2} \theta+\right. \\
& \left.I_{f y} \cos ^{2} \theta\right) \ddot{\varphi}+I_{p} \Omega \dot{\theta} \cos \theta+m_{s} g L \cos \theta \sin \varphi=0
\end{aligned}
$$

Neglecting the terms of a higher power from the Eqs. (11)(13) for small vibrations and substituting $\sin \theta=\theta, \cos \theta=1$, $\sin \varphi=\varphi$, and $\cos \varphi=1$, the equations can be reduced to:

$$
\begin{aligned}
& M_{t} \ddot{y}+\ddot{\theta} m_{s} L+k(y-Z)=0 \\
& \left(I_{o}+I_{f z}+m_{s} L^{2}\right) \ddot{\theta}+\ddot{y} m_{s} L-I_{p} \Omega \dot{\varphi}+m_{s} g L \theta=0 \\
& \left(m_{s} L^{2}+I_{p y}+I_{o}+I_{f y}\right) \ddot{\varphi}+I_{p} \Omega \dot{\theta}+m_{s} g L \varphi=0 .
\end{aligned}
$$

Therefore, the natural frequencies of the primary mass $\left(M_{t}\right)$, the inner gimbal and the outer gimbal can be determined as 


$$
\begin{aligned}
& {\left[\begin{array}{ccc}
-\omega^{2} M_{t}+k & -\omega^{2} m_{s} L & 0 \\
-\omega^{2} m_{s} L & -\omega^{2}\left(I_{o}+I_{f z}+m_{s} L^{2}\right)+m_{s} g L & -j \omega I_{p} \Omega \\
0 & j \omega I_{p} \Omega & -\omega^{2}\left(m_{s} L^{2}+I_{p y}+I_{o}+I_{f y}\right)+m_{s} g L
\end{array}\right]\left\{\begin{array}{l}
y_{0} \\
\theta_{0} \\
\varphi_{0}
\end{array}\right\}=\left\{\begin{array}{c}
k z_{0} \\
0 \\
0
\end{array}\right\} .} \\
& \Omega= \pm \frac{\sqrt{\left[-\omega^{2}\left(I_{o}+I_{f z}+m_{s} L^{2}\right)+m_{s} g L\right]\left[-\omega^{2}\left(m_{s} L^{2}+I_{p y}+I_{o}+I_{f y}\right)+m_{s} g L\right]}}{I_{p} \omega} . \\
& \left(\Omega I_{p}\right)_{\mathrm{opt}}= \pm \frac{\sqrt{\left(\omega_{n}^{2}-\omega_{i g}^{2}\right)\left(\omega_{n}^{2}-\omega_{o g}^{2}\right)\left(I_{o}+I_{f z}+m_{s} L^{2}\right)\left(m_{s} L^{2}+I_{p y}+I_{o}+I_{f y}\right)}}{\omega_{n}} .
\end{aligned}
$$

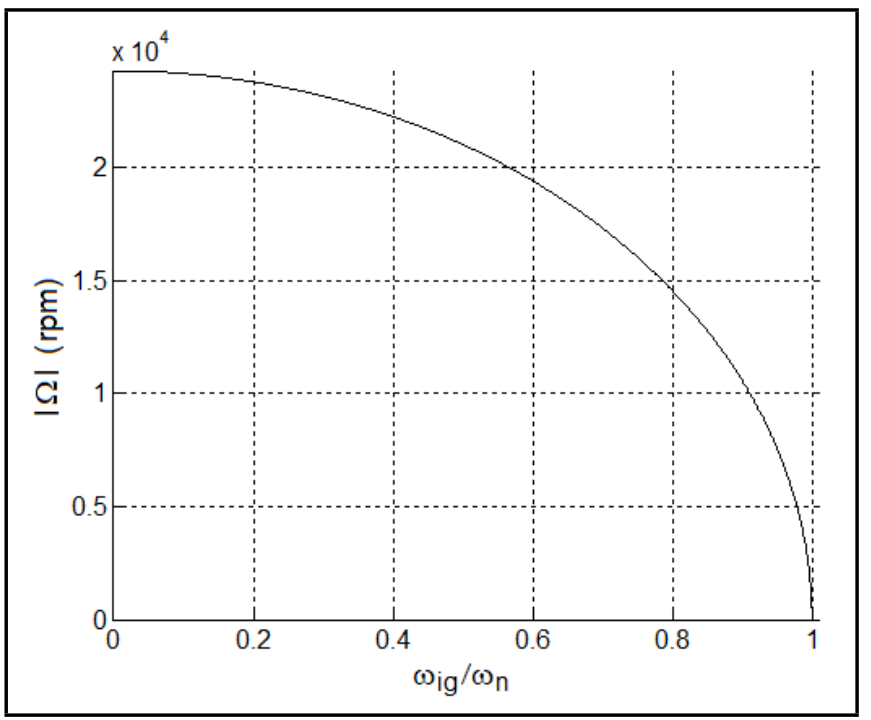

Figure 4. Effect of the disk speed $\Omega$ on the response of the primary mass $M_{t}$.

follows, respectively:

$$
\begin{aligned}
\omega_{n} & =\sqrt{\frac{k}{M_{t}}} ; \\
\omega_{i g} & =\sqrt{\frac{m_{s} g L}{I_{o}+I_{f z}+m_{s} L^{2}}} ; \\
\omega_{o g} & =\sqrt{\frac{m_{s} g L}{I_{p y}+I_{o}+I_{f y}+m_{s} L^{2}}} .
\end{aligned}
$$

\subsection{Frequency Equations and Optimal Tuning}

When the harmonic disturbing base excitation is $Z=$ $z_{0} e^{j \omega t}$, the responses may be written as an angular frequency $\omega$ :

$$
y=y_{0} e^{j \omega t}, \quad \theta=\theta_{0} e^{j \omega t}, \quad \text { and } \varphi=\varphi_{0} e^{j \omega t} .
$$

Substitution of Eq. (18) into Eqs. (14)-(16) gives in the matrix form of Eq. (19).

By equating the determinant of the coefficients of $y_{0}, \theta_{0}$, and $\varphi_{0}$ to zero, we obtain the characteristic equation as:

$$
a_{0}\left(\lambda^{3}+a_{1} \lambda^{2}+a_{2} \lambda+a_{3}\right)=0
$$

in which:

$$
\begin{aligned}
\lambda= & \omega^{2} ; \\
a_{0}= & \left(m_{s} L\right)^{2}\left(m_{s} L^{2}+I_{p y}+I_{o}+I_{f y}\right)- \\
& M_{t}\left(I_{0}+I_{f z}+m_{s} L^{2}\right)\left(m_{s} L^{2}+I_{p y}+I_{o}+I_{f y}\right) ; \\
a_{1}= & {\left[M_{t} m_{s} g L\left(2 m_{s} L^{2}+2 I_{o}+I_{p y}+I_{f y}+I_{f z}\right)+\right.} \\
& k\left(I_{o}+I_{f z}+m_{s} L^{2}\right)\left(m_{s} L^{2}+I_{p y}+I_{o}+I_{f y}\right)+ \\
& \left.M_{t}\left(I_{p} \Omega\right)^{2}-\left(m_{s} L\right)^{2} m_{s} g L\right] / a_{0} ; \\
a_{2}= & {\left[-M_{t}\left(m_{s} g L\right)^{2}-k m_{s} g L\left(2 m_{s} L^{2}+2 I_{o}+I_{p y}+\right.\right.} \\
& \left.\left.I_{f y}+I_{f z}\right)-k\left(I_{p} \Omega\right)^{2}\right] / a_{0} ; \\
a_{3}= & k\left(m_{s} g L\right)^{2} / a_{0} .
\end{aligned}
$$

The roots of this equation are: ${ }^{16}$

$$
\begin{aligned}
& \lambda_{1}=-\frac{1}{2}(S+T)-\frac{1}{3} a_{1}+\frac{1}{2} j \sqrt{3}(S-T) ; \\
& \lambda_{2}=-\frac{1}{2}(S+T)-\frac{1}{3} a_{1}-\frac{1}{2} j \sqrt{3}(S-T) ; \\
& \lambda_{3}=S+T-\frac{1}{3} a_{1} ;
\end{aligned}
$$

in which:

$$
\begin{aligned}
Q & =\frac{3 a_{2}-a_{1}^{2}}{9} ; \\
R & =\frac{9 a_{1} a_{2}-27 a_{3}-2 a_{1}^{3}}{54} ; \\
S & =\sqrt[3]{R+\sqrt{Q^{3}+R^{2}}} ; \\
T & =\sqrt[3]{R-\sqrt{Q^{3}+R^{2}}}
\end{aligned}
$$

Besides, Eq. (19) can be solved for $y_{0}, \theta_{0}$, and $\varphi_{0}$ as:

$$
\begin{aligned}
\frac{y_{0}}{z_{0}}= & k\left\{[ - \omega ^ { 2 } ( I _ { o } + I _ { f z } + m _ { s } L ^ { 2 } ) + m _ { s } g L ] \left[-\omega^{2}\left(m_{s} L^{2}+\right.\right.\right. \\
& \left.\left.\left.I_{p y}+I_{o}+I_{f y}\right)+m_{s} g L\right]-\left(I_{p} \Omega \omega\right)^{2}\right\} /\left\{a _ { 0 } \left(\lambda^{3}+\right.\right. \\
& \left.\left.a_{1} \lambda^{2}+a_{2} \lambda+a_{3}\right)\right\} \\
\theta_{0}= & \frac{\omega^{2} z_{0} m_{s} L k\left[-\omega^{2}\left(m_{s} L^{2}+I_{p y}+I_{o}+I_{f y}\right)+m_{s} g L\right]}{a_{0}\left(\lambda^{3}+a_{1} \lambda^{2}+a_{2} \lambda+a_{3}\right)} \\
\left|\varphi_{0}\right|= & \frac{\omega^{3} z_{0} k m_{s} L I_{p} \Omega}{a_{0}\left(\lambda^{3}+a_{1} \lambda^{2}+a_{2} \lambda+a_{3}\right)} .
\end{aligned}
$$




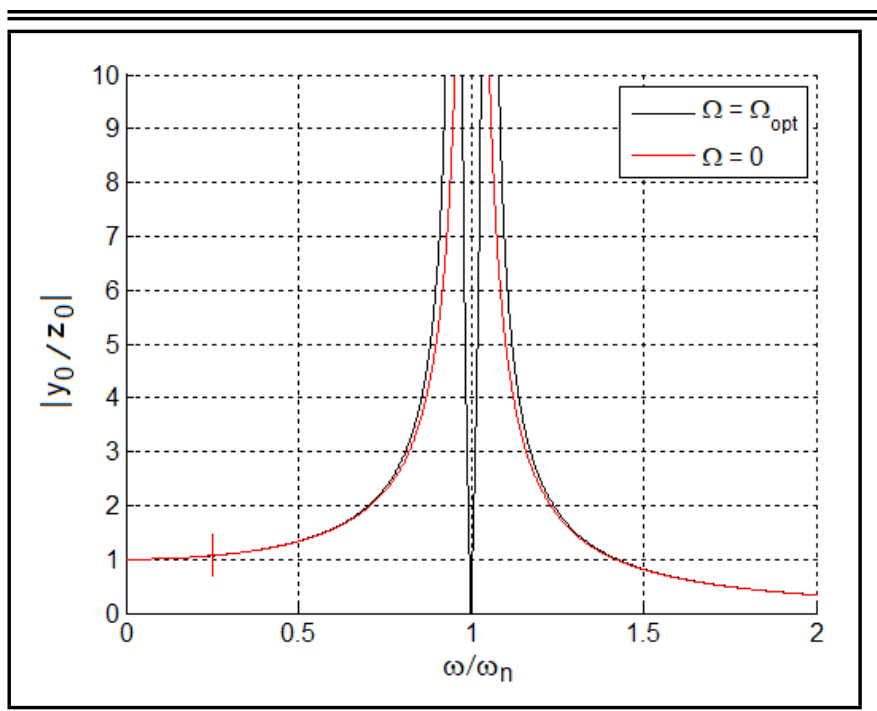

Figure 5. Effect of a gyro-pendulum absorber on the response of the primary mass $M_{t}$.

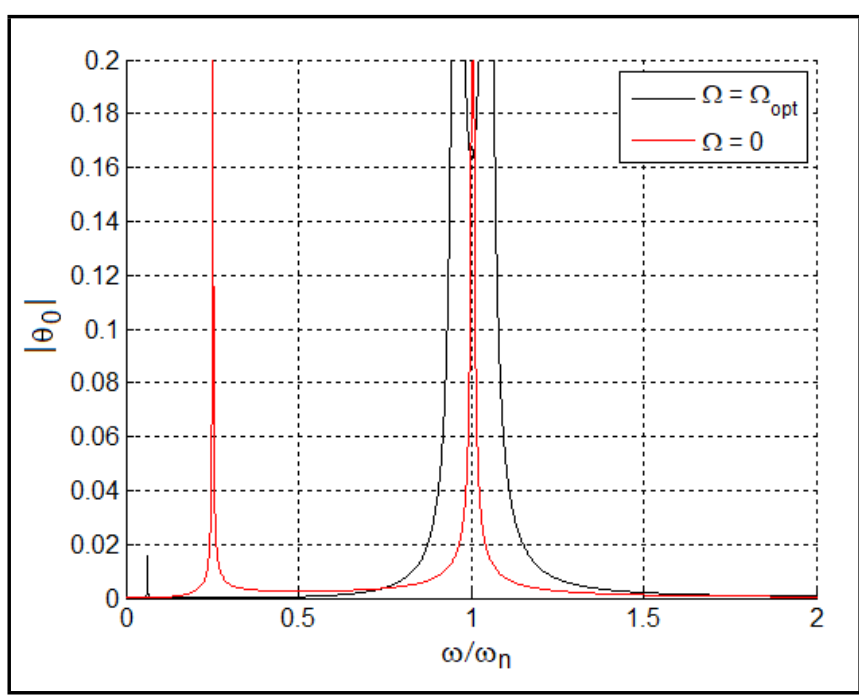

Figure 6. The response $\theta_{0}$ of a gyro-pendulum absorber.

In order to make the displacement of the primary mass zero, the numerator of Eq. (24) should be set as:

$$
\begin{gathered}
k\left\{[ - \omega ^ { 2 } ( I _ { o } + I _ { f z } + m _ { s } L ^ { 2 } ) + m _ { s } g L ] \left[-\omega^{2}\left(m_{s} L^{2}+I_{p y}+\right.\right.\right. \\
\left.\left.\left.I_{o}+I_{f y}\right)+m_{s} g L\right]-\left(I_{p} \Omega \omega\right)^{2}\right\}=0 .
\end{gathered}
$$

Thus, the disk speed is yielded by Eq. (28).

It can be seen from Eq. (28) and Fig. 4 that the optimum disk speed is equal to zero when the natural frequency of the inner pendulum is equal to the resonant frequency. However, this equilibrium is not needed anymore with an adjustable disk speed of the gyroscope to obtain zero amplitude for the target frequency. From Eq. (28), the calculated optimum disk speed $\Omega$ for $\omega=\omega_{n}$ is $23373 \mathrm{rpm}$. However, optimum disk speed is about $22500 \mathrm{rpm}$ from the numerical solution of Eqs. (7)-(9). The reason for this $3.88 \%$ error is due to the fact that the linearized equations reduced the accuracy of the approximation.

The responses of the system as a function of the frequency ratio $\omega / \omega_{n}$ are shown in Figs. 5, 6, and 7. When the optimum disk speed is used, the gyro-pendulum absorber includes two resonant frequencies $\left(\omega_{2}\right.$ and $\left.\omega_{3}\right)$ at which the response is

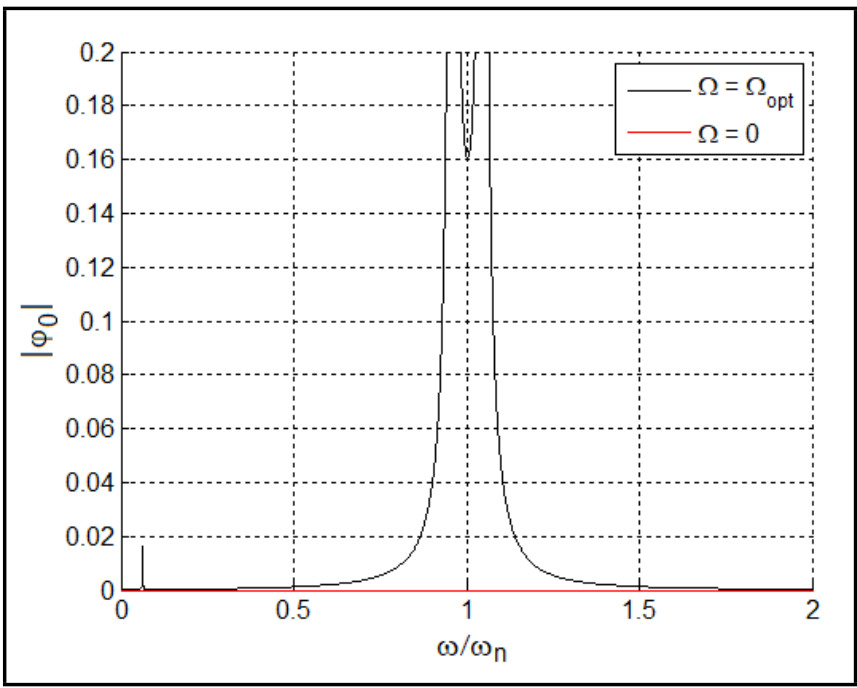

Figure 7. The response $\varphi_{0}$ of a gyro-pendulum absorber.

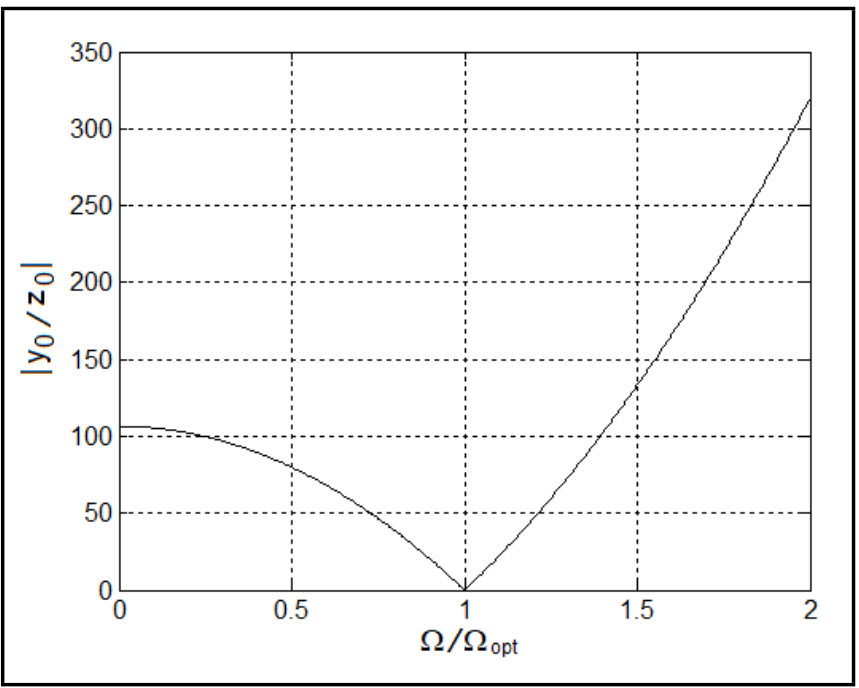

Figure 8. Effect of the disk speed $\Omega$ on the response of the primary mass $M_{t}$.

characteristic of two-degree-of-freedom systems excited by a harmonic excitation. While eliminating vibration at the known impressed frequency $\omega$, one of the system's two natural frequencies is less than the tuned frequency while the other is greater. So, the excitation frequency must be kept away from the resonant frequencies $\left(\omega_{2}\right.$ and $\left.\omega_{3}\right)$.

The response of the primary mass as functions of the disk speed ratio $\Omega / \Omega_{\text {opt }}$ is shown in Fig. 8 for the value of the primary mass frequency $\omega_{n}$. It can be seen that the amplitude of the primary mass is zero when the disk speed $(\Omega)$ is tuned to the optimum speed $\left(\Omega_{\mathrm{opt}}\right)$. If the gyro operates at other speeds, the amplitude of vibration of the primary mass becomes larger.

\section{RESULTS}

In the following calculations, Lagrange's equations of motion may be solved by using a Matlab software tool that involves the fourth-order Runge-Kutta method. The physical parameters of the numerical examples are given in Table 1. In order to identify the dynamical behaviour, time response was simulated with the time step size of $0.005 \mathrm{~s}$, and zero initial conditions. 


\begin{tabular}{|c|c|c|c|c|c|c|c|c|c|}
\hline $\begin{array}{c}\Omega \\
(\mathrm{rpm}) \\
\end{array}$ & $\begin{array}{c}\text { Theoretical } \omega_{1} \\
(\mathrm{~Hz})\end{array}$ & $\begin{array}{c}\text { ANSYS } \omega_{1} \\
(\mathrm{~Hz})\end{array}$ & $\begin{array}{c}\text { Error for } \omega_{1} \\
\%\end{array}$ & $\begin{array}{c}\text { Theoretical } \omega_{2} \\
(\mathrm{~Hz})\end{array}$ & $\begin{array}{c}\text { ANSYS } \omega_{2} \\
(\mathrm{~Hz})\end{array}$ & $\begin{array}{c}\text { Error for } \omega_{2} \\
\%\end{array}$ & $\begin{array}{c}\text { Theoretical } \omega_{3} \\
(\mathrm{~Hz})\end{array}$ & $\begin{array}{c}\text { ANSYS } \omega_{3} \\
(\mathrm{~Hz})\end{array}$ & $\begin{array}{c}\text { Error for } \omega_{3} \\
\%\end{array}$ \\
\hline 0 & 0.5898 & 0.6087 & 3.1050 & 0.5965 & 0.6171 & 3.3382 & 2.3793 & 2.3793 & 0.0000 \\
\hline 5000 & 0.4015 & 0.4200 & 4.4048 & 0.8760 & 0.8940 & 2.0134 & 2.3799 & 2.3799 & 0.0000 \\
\hline 10000 & 0.2850 & 0.3007 & 5.2212 & 1.2330 & 1.2477 & 1.1782 & 2.3818 & 2.3819 & 0.0042 \\
\hline 15000 & 0.2147 & 0.2276 & 5.6678 & 1.6334 & 1.6444 & 0.6689 & 2.3870 & 2.3872 & 0.0084 \\
\hline 20000 & 0.1701 & 0.1808 & 5.9181 & 2.0452 & 2.0525 & 0.3557 & 2.4067 & 2.4074 & 0.0291 \\
\hline 30000 & 0.1186 & 0.1264 & 6.1709 & 2.3518 & 2.3519 & 0.0043 & 3.0012 & 3.0034 & 0.0733 \\
\hline 35000 & 0.1027 & 0.1095 & 6.2100 & 2.3594 & 2.3594 & 0.0000 & 3.4543 & 3.4534 & 0.0261 \\
\hline 40000 & 0.0905 & 0.0965 & 6.2176 & 2.3625 & 2.3624 & 0.0042 & 3.9156 & 3.9112 & 0.1125 \\
\hline 45000 & 0.0808 & 0.0862 & 6.2645 & 2.3641 & 2.3640 & 0.0042 & 4.3809 & 4.3726 & 0.1898 \\
\hline 50000 & 0.0730 & 0.0779 & 6.2901 & 2.3651 & 2.3650 & 0.0042 & 4.8487 & 4.8359 & 0.2647 \\
\hline
\end{tabular}

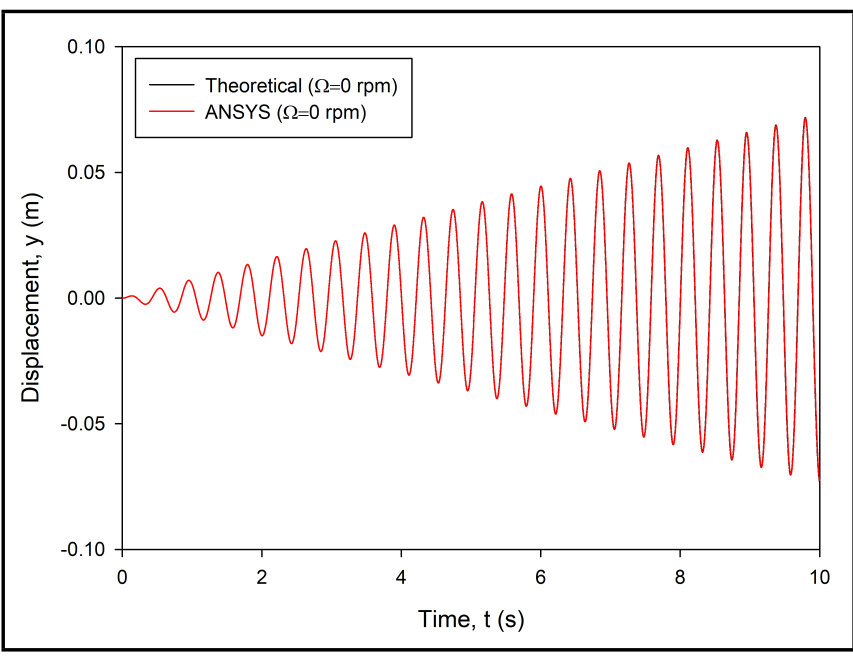

Figure 9. Comparison of the theoretical and ANSYS results without disk speed. The period is the same as the natural period of the primary mass $M_{t}$ with a base excitation $Z=z_{0} \cos \left(\omega_{n} t\right) .\left(z_{0}=0.001 \mathrm{~m}\right.$ and $\omega_{n}=$ $2.3682 \mathrm{~Hz})$.

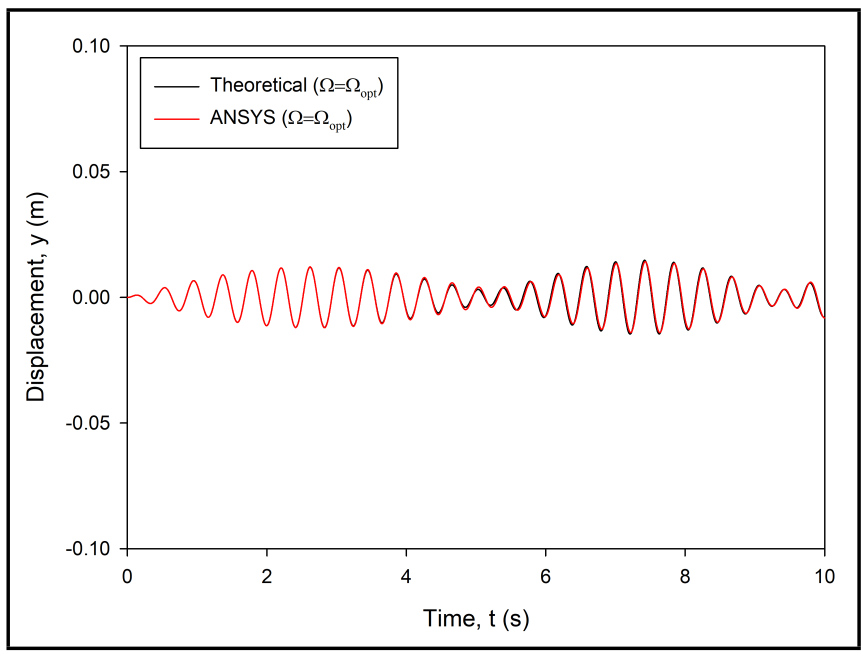

Figure 10. Comparison of the theoretical and ANSYS results with optimum disk speed. The period is the same as the natural period of the primary mass $M_{t}$ with a base excitation $Z=z_{0} \cos \left(\omega_{n} t\right) . \quad\left(z_{0}=0.001 \mathrm{~m}, \Omega_{\mathrm{opt}}=\right.$ $22500 \mathrm{rpm}$ and $\omega_{n}=2.3682 \mathrm{~Hz}$ ).

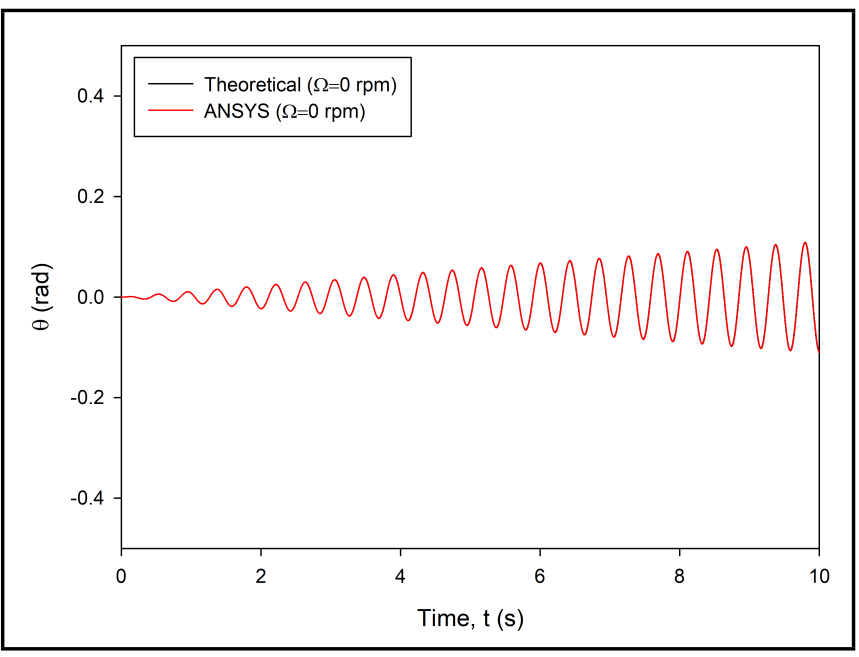

Figure 11. Comparison of the theoretical and ANSYS results without disk speed. The period is the same as the natural period of the primary mass $M_{t}$ with a base excitation $Z=z_{0} \cos \left(\omega_{n} t\right) .\left(z_{0}=0.001 \mathrm{~m}\right.$ and $\omega_{n}=$ $2.3682 \mathrm{~Hz})$.

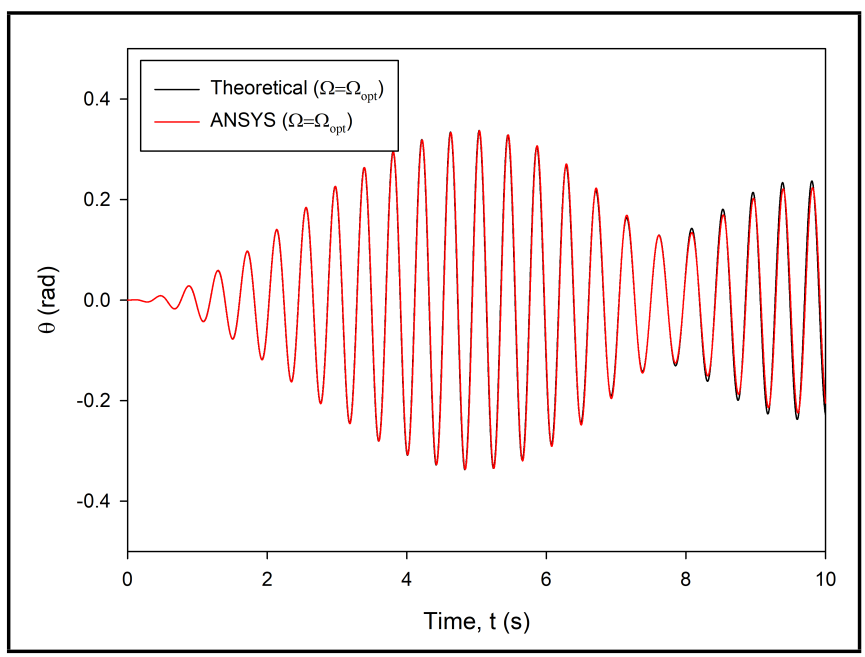

Figure 12. Comparison of the theoretical and ANSYS results with optimum disk speed. The period is the same as the natural period of the primary mass $M_{t}$ with a base excitation $Z=z_{0} \cos \left(\omega_{n} t\right) . \quad\left(z_{0}=0.001 \mathrm{~m}, \Omega_{\mathrm{opt}}=\right.$ $22500 \mathrm{rpm}$ and $\omega_{n}=2.3682 \mathrm{~Hz}$ ). 


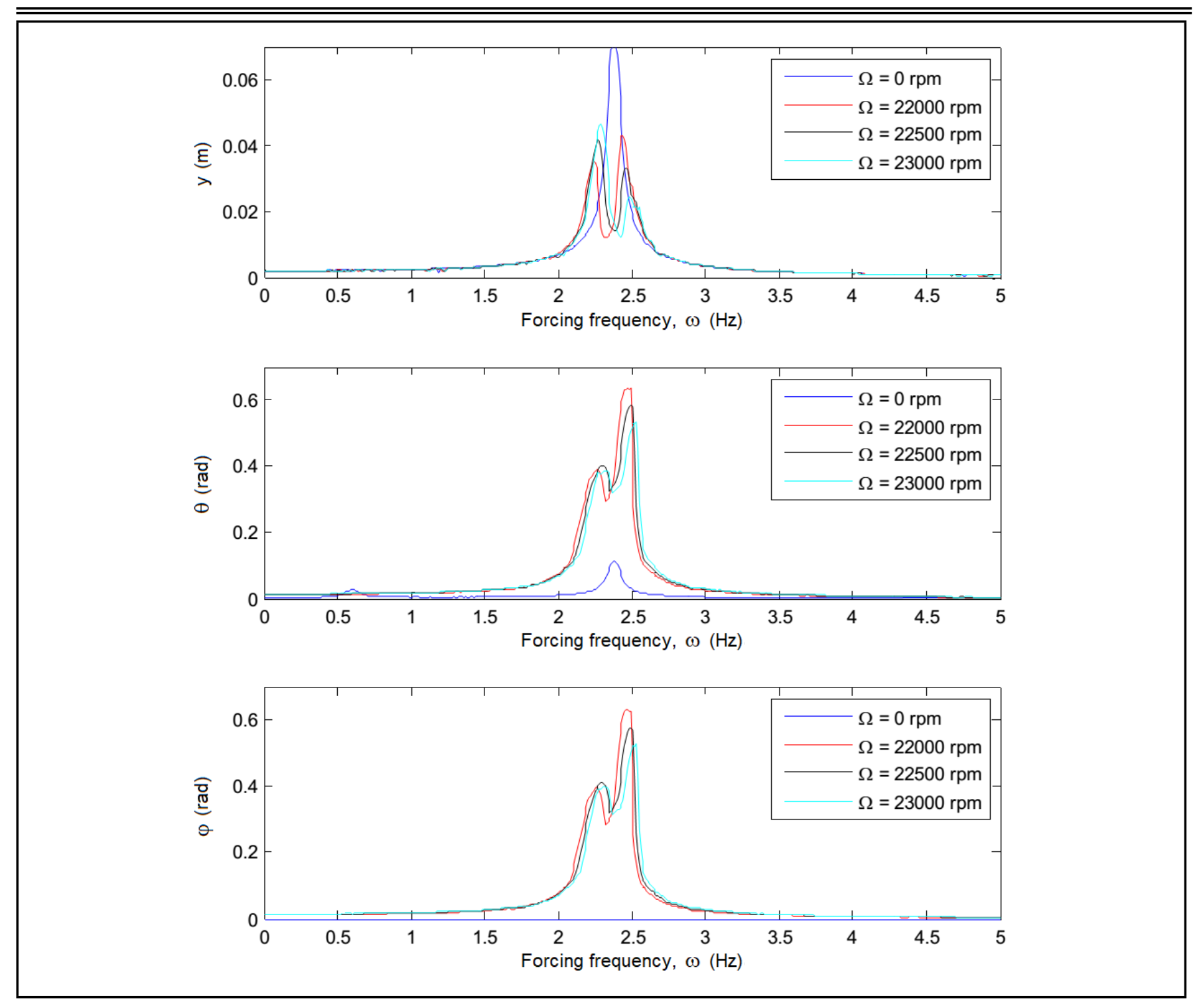

Figure 13. Theoretical frequency response curves of the primary mass and the gyro-pendulum for different disk speeds $(\Omega)$ with a base excitation $Z=$ $z_{0} \cos \left(\omega_{n} t\right) .\left(z_{0}=0.001 \mathrm{~m}\right.$ and $\left.\omega_{n}=2.3682 \mathrm{~Hz}\right)$.

In this section, the calculated results of Lagrange's equations were compared with the results obtained from ANSYS Workbench to work out its displacements and mode frequencies. The simplified dynamic simulation model is given in Fig. 2. The theoretical and ANSYS results of the resonant frequencies with different disk speeds of gyro are given in Table 2. It can be deduced from the comparison that the tendencies of the mode frequencies agreed well with the ANSYS ones. However, the first mode frequencies $\left(\omega_{1}\right)$ contain minor errors due to the linearized equations. When rotor speed has higher values, which means more force in the horizontal axis, the mode frequencies of $\omega_{2}$ and $\omega_{3}$ increase while the first mode frequency $\omega_{1}$ decreases.

The time responses of displacements from the theoretical and ANSYS approaches are shown in Fig. 9-12. The comparison showed that the displacements of the theoretical and ANSYS results are almost the same for the value of the primary mass frequency $\omega_{n}$. Meanwhile, the vibration mitigation performance of the gyro-pendulum increased using the optimum rotational speed of disk at the resonant frequency of primary mass.
Figure 13 represents the frequency response curves of different disk speeds $(\Omega)$ for the frequency of primary mass $\left(M_{t}\right)$. The gyro-pendulum has significantly reduced the vibration at the frequency $\omega_{n}=2.3682 \mathrm{~Hz}$. However, two resonant peaks arise at the mode frequencies, $\omega_{2}$ and $\omega_{3}$, while eliminating vibration at the frequency of primary mass. At a certain speed, there is significant mitigation in the vibration for $\omega_{n}=2.3682 \mathrm{~Hz}$. So, the displacement response of the primary mass can be considerably reduced when the optimum disk speed is used.

\section{CONCLUSIONS}

This paper provides some theoretical equations to investigate a gyro-pendulum absorber which can be capable of producing unidirectional thrust along the forcing excitation axis while the disk is spinning. In order to verify the above results, the model is built strictly according to the size in Table 1. Therefore, the results of ANSYS are exactly in accord with theoretical equations due to the simplified model. The correctness of the theoretical results is verified by ANSYS simulations. From the consistency of the present study, the following 
conclusions are found:

- It can be deduced from the comparison that the theoretical results agree well with the ANSYS ones. The resonant frequencies calculated by the proposed theoretical prediction match well with those obtained by ANSYS.

- This paper introduces the frequency equations to investigate the optimum disk speed of the gyro. The study clearly indicates that the relation between the gyropendulum and the resonant frequency of primary mass should be determined by Eq. (29), which is an approximation of the optimum angular momentum of the gyropendulum. This equation shows that, as much as disk speed is increased, the rotary inertia of the disk can be reduced. Since the rotary inertia of the disk is proportional to the mass of disk, the weight and volume of the system can be reduced. Additionally, the rotation of disk can be used in both directions, in which the pendulum rotates in direction of the disk when the rotary inertia of the disk is assumed to be positive.

The proposed gyro-pendulum has a practical advantage in comparison with conventional ones because, with an adjustable disk speed of gyroscope, the gyro-pendulum is efficient on a large frequency range from the pendulum resonance to the main system resonance.

\section{REFERENCES}

1 Ibrahim, R. Recent advances in nonlinear passive vibration isolators, Journal of Sound and Vibration, 314, 371-452, (2008). https://dx.doi.org/10.1016/j.jsv.2008.01.014

2 Cuvalci, O. The effect of detuning parameters on the absorption region for a coupled system: a numerical and experimental study, Journal of Sound and Vibration, 229 (4), 837-857, (2000). https://dx.doi.org/10.1006/jsvi.1999.2526

3 Ertas, A., Cuvalci, O., and Ekwaro-Osire S. Performance of pendulum absorber for a nonlinear system of varying orientation, Journal of Sound and Vibration, 229 (4), 913-933, (2000). https://dx.doi.org/10.1006/jsvi.1999.2521

4 Soong, T. T. and Dargush, G. F. Passive energy dissipation system in structural engineering, Wiley, New York, NY, USA, (1997).

5 Frahm, H. Device for damping vibrations of bodies, U.S.Patent No.989,958,1911.

6 Chung, J. T. Vibration absorber for reduction of the inplane vibration in an optical disk drive, IEEE Transactions on Consumer Electronics, 50 (2), 552-557, (2004). https://dx.doi.org/10.1109/TCE.2004.1309422
$7 \mathrm{Wu}, \mathrm{T}$. X. On the railway track dynamics with rail vibration absorber for noise reduction, Journal of Sound and Vibration, 309 (3-5), 739-755, (2008). https://dx.doi.org/10.1016/j.jsv.2007.07.049

8 Webster, A. C. and Vaicaitis, R. Application of tuned mass dampers to control vibrations of composite floor systems, Engineering Journal-American Institute of Steel Construction Inc, 29 (3), 116-124, (1992).

9 Mori, H., Mikhyeyev, O., Nagamine, T., Mori, M., and Sato, Y. Effect of a dynamic absorber on frictioninduced vibration of a rectangular plate, Journal of $\mathrm{Me}$ chanical Science and Technology, 24 (1), 93-96, (2010). https://dx.doi.org/10.1007/s12206-009-1168-8

10 Viet, L. D. and Park, Y. Vibration control of the axisymmetric spherical pendulum by dynamic vibration absorber moving in radial direction, Journal of Mechanical Science and Technology, 25 (7), 1703-1709, (2011). https://dx.doi.org/10.1007/s12206-011-0418-8

11 Ormondroyd, J. and DenHartog, J. P. The theory of the dynamic vibration absorber, ASME Journal of Applied Mechanics, 50 (7), 9-22, (1928).

12 Nagase, T. and Hisatoku, T. Tuned pendulum mass damper installed in crystal tower, The Structural Design of Tall Buildings, 1, 35-56, (1992). https://dx.doi.org/10.1002/tal.4320010105

13 Ünker, F. and Çuvalcı, O. Optimum tuning of a gyroscopic vibration absorber for vibration control of a vertical cantilever beam with tip mass, International Journal of Acoustics \& Vibration, 24 (2), 210-216, (2019). https://dx.doi.org/10.20855/ijav.2019.24.21158

14 Ünker, F. and Çuvalcı, O. Seismic motion control of a column using a gyroscope, In Procedia-Social and Behavioral Sciences, 195, 2316-2325, (2015). https://dx.doi.org/10.1016/j.sbspro.2015.06.183

15 Viet, L. D. A single mass dynamic vibration absorber producing linear damping, Coriolis damping and gyro-damping, Proceedings of the Institution of Mechanical Engineers, Part C: Journal of Mechanical Engineering Science, 230 (4), 506-513, (2016). https://dx.doi.org/10.1177/0954406215618227

16 Spiegel, M. R. Mathematical handbook of formulas and tables, Schaum's outline series, McGraw-Hill Education, 4th edition, (2012). 